This item was submitted to Loughborough's Research Repository by the author.

Items in Figshare are protected by copyright, with all rights reserved, unless otherwise indicated.

\title{
Metallic inclusions in a non-uniform lattice
}

PLEASE CITE THE PUBLISHED VERSION

http://dx.doi.org/10.1109/LAPC.2011.6114148

PUBLISHER

Loughborough University (@ IEEE)

VERSION

AM (Accepted Manuscript)

LICENCE

CC BY-NC-ND 4.0

REPOSITORY RECORD

Njoku, Chinwe C., W.G. Whittow, J.C. Vardaxoglou, Chris Toprakcioglu, and K.-A.Th. Thoma. 2019. "Metallic Inclusions in a Non-uniform Lattice". figshare. https://hdl.handle.net/2134/9990. 
This item was submitted to Loughborough's Institutional Repository (https://dspace.lboro.ac.uk/) by the author and is made available under the following Creative Commons Licence conditions.

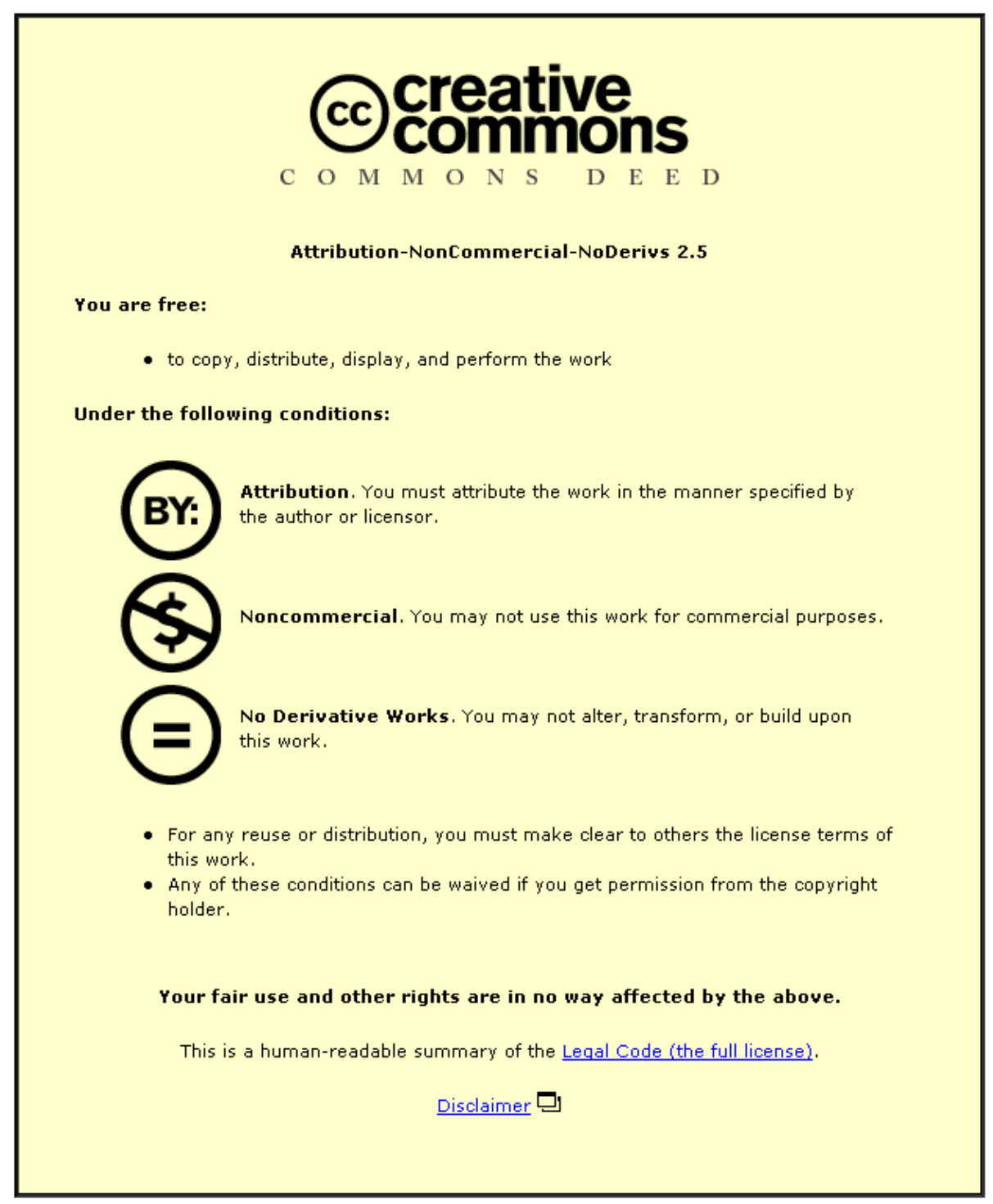

For the full text of this licence, please go to: http://creativecommons.org/licenses/by-nc-nd/2.5/ 


\title{
Metallic Inclusions in a Non-Uniform Lattice
}

\author{
Chinwe C. Njoku ${ }^{\# 1}$, William G. Whittow ${ }^{\# 2}$, Yiannis C.Vardaxoglou ${ }^{\# 3}$, Chris Toprakcioglu ${ }^{* 4}$, K.-A. Th. Thoma ${ }^{* 5}$ \\ ${ }^{\#}$ Department of Electrical \& Electronic Engineering, Loughborough University, UK \\ *Department of Physics, University of Patras, Patras 26500, Greece. \\ ${ }^{1}$ c.c.njoku@lboro.ac.uk \\ ${ }^{2}$ w.g.whittow@lboro.ac.uk \\ jj.c.vardaxoglou@lboro.ac.uk \\ ${ }^{4}$ ctop@physics.upatras.gr \\ ${ }^{5}$ phaethon@physics.upatras.gr
}

\begin{abstract}
This paper is part of a larger project which aims to investigate the fabricational and electromagnetic advantages of creating integrated antenna systems using emerging nanomanufacturing technology. It has been known for several decades that the effective permittivity of a mixture consisting of dielectric inclusions in a host dielectric can be controlled by varying the permittivity, size and spacing of the inclusions. Various authors have developed theoretical equations to analyse these structures but are typically limited to spherical inclusions in a uniform cubic lattice. This paper extends this work from spheres in a uniform mesh to investigate thin metallic inclusions in a dielectric host using a non-uniform lattice (spacing in $x, y$ and $z$, not the same). Electromagnetic simulations of these structures have been compared to canonical equations of spheres in a cubic uniform lattice with the same volume ratio.
\end{abstract}

\section{INTRODUCTION}

The creation of novel substrates with high permittivity values using low-cost materials will provide antenna and material engineers a variety of dielectric and magneto dielectric substrates that have the potential of being custommade. These synthesized materials can be formed from homogenous materials within which are embedded inclusions of different sizes and in different lattice arrangements, typically cubic. The sizes and arrangement of these inclusions are dependent on the specific requirements. This potential to manipulate existing materials has prompted extensive research [1]-[11] into the creation of these materials that provide the electromagnetic (EM) properties, losses and other parameters required for the system to have. Related research has been in the areas of metamaterials [5], artificial materials [7] and double negative materials [9], [10]. Nano-technology fabrication techniques can be used in the creation of these heterogeneous structures [12]-[14], by using additive manufacturing processes as opposed to destructive processes, such as electrodeposition and self-assembly. Complete antenna structures and associated RF circuitry (as shown in Fig. 1) are possible by strategically placing metallic and insulating elements. The ability to control the particles' volume ratio allows for the creation of novel and custommade substrates. Faster build processes and reduced production costs are potential advantages of this nanofabrication method. Material cost reductions are expected to become significant as the world consumes more raw resources and this is particularly true as the average cost of dielectric materials increases with the increase in their permittivity values. In addition, EM advantages relative to bandwidth, size and efficiency, can be achieved by different methods.

In [15], we show what our intended antenna structure might be in the long run and not what has been achieved already. In the diagram, all the components of the antenna system are made from smaller particles.

The aim of this paper is to present our investigations of heterogeneous media with emphasis on their use as substrates in microwave designs. In Section II, we give a brief account of relevant equations from reviewed literature on the effective EM properties of infinite heterogeneous media. We also present a "resonant inverse scattering" method from [16] of extracting the $\varepsilon$ and $\mu$ of a material from the scattering (S-) parameters obtained from 3D Finite Difference Time Domain (FDTD) simulations using Empire $\mathrm{XCcel}^{\mathrm{TM}}$. A detailed review was presented in [15], [17]. In Section III, an overview on the determination of the dielectric constants of metals such as Copper, using the Drude model [18], [19] is presented. A look at a uniform (that is, equal spacing in the $\mathrm{x}, \mathrm{y}$ and $\mathrm{z}$ directions) and non-uniform (in only the $\mathrm{x}$ direction) heterogeneous structure having Copper inclusions is also presented. Conclusions are made in Section in IV.

Small-sized dielectric inclusions having a higher permittivity and/or permeability can be added to a low dielectric, homogenous host to increase the effective electromagnetic (EM) properties of the final mixture. This has been proven both theoretically and via measurements. In our previous papers, we have reviewed such literatures and presented simulations for comparisons. In order to cause a significant increase in the effective permittivity of the mixture, metallic or conducting small-sized inclusions can also be added [1], [6], [20]-[22]. Note, that in this paper the permittivity and permeability values are the relative values and should be scaled by the free space values. 


\section{THEORETICAL AND EM SiMULATION ANALYSIS}

The theories established in [1] form the general basis for the study of heterogeneous substrates/artificial dielectrics, although the earliest study is in [2]. The Clausius-Mossoti equation is used in [4]-[9] for describing the effective EM properties of a heterogeneous material. However, this equation is represented differently in the literature as: some exclude the EM properties of the host [5]; only examine the static DC case and have no frequency terms [7][8]; treat the particles' densities and polarisabilities differently in electric and magnetic modes [9] or does not distinguish between the inclusion's EM properties on a small or bulk scale [8]. They are similar in that their analyses are based on an infinite or semi-infinite medium. In general, an expression for the effective EM properties of a heterogeneous medium is given in [4] as

$$
K_{\mathrm{eff}}=K_{1} \frac{\left[\left(K_{2}+2 K_{1}\right)\left(K_{1}-K_{2}\right)^{-1}\right]-2 p+C\left(K_{1}, K_{2}, p\right)}{\left[\left(K_{2}+2 K_{1}\right)\left(K_{1}-K_{2}\right)^{-1}\right]+p+C\left(K_{1}, K_{2}, p\right)}
$$

where $K_{1}, K_{2}$ and $K_{\text {eff }}$ are the appropriate EM parameters of the host material, the inclusions and the mixture respectively; $p$ is the volume fraction of the inclusions, which is based on the local unit cell of the medium. $C\left(K_{1}, K_{2}, p\right)$ represents corrections for higher-order multipole terms as a result of the scattered fields [1], [23].

3D FDTD simulations have been used to simulate heterogeneous media to allow for finite and/or asymmetric structures. The effective $\varepsilon$ and $\mu$ of a medium can be obtained from the S-parameters as shown in [16], [24]-[26]. The Sparameters obtained were used in an Inversion process [16] to obtain the effective $\varepsilon$ and $\mu$ of the simulated structure. This also allows comparison with the results from the canonical analysis. The infinite medium assumption from the equations was replicated in the simulations with the use of Perfect Electric and Magnetic Conductor (PEC and PMC) boundaries on the two axes forming the plane of incidence of the plane wave impinging on the structure. The structure was finite along the axis of propagation.

In the equations and simulations, cubical lattices are used, that is, the inclusions are at the same distance from each other in all axes. Also, spherical inclusions are used in both.

\section{A. Theoretical Results}

Graphical results using [1], [5], [6], [9] are presented in Fig. 1 for dielectric inclusions. Data used: particle's size, $a=50$ $\mu \mathrm{m}$, particle size, $s=105 \mu \mathrm{m}, \varepsilon_{2}=10.21, \mu_{1}=\mu_{2}=1$, and $\varepsilon_{1}=2.08$.

Note, the equations in [5] do not include the host permittivity value and are accurate when the host is close to air but caution should be applied when using the equations in [5] to calculate effective permittivities where the host is greater than 1. Fig. 1 shows that the other equations from the literature agree over the frequency range considered. The average effective permittivity, $\varepsilon_{\text {eff }}$ of the heterogeneous structure from the canonical equations is 4.23 .

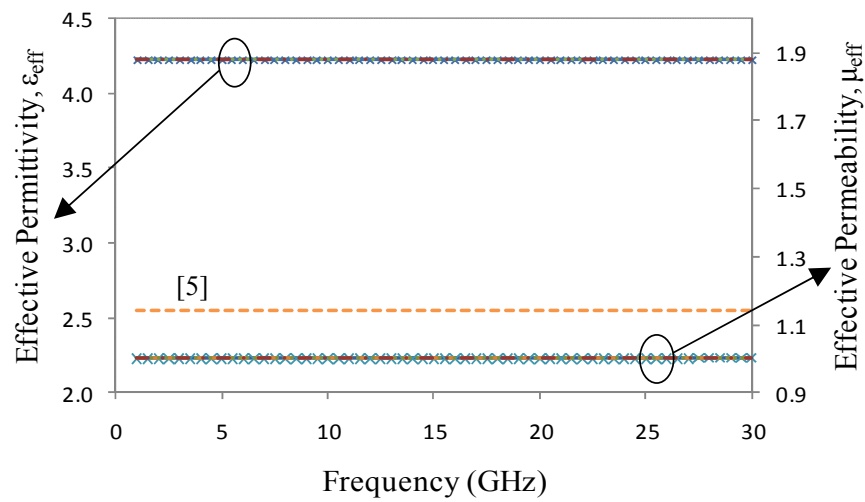

Fig. 1. Variation of effective $\varepsilon$ and $\mu$ of a heterogeneous medium with frequency for $50 \mu \mathrm{m}$ spheres, using canonical analysis by [1] (-), [5] (- ), $[6](\cdots),[9](-\cdot-)$.

\section{B. FDTD Results}

To compare the theoretical equations with electromagnetic simulations, the heterogeneous sample was placed in a transmission line. By using magnetic and electric boundaries the samples are infinite in directions perpendicular to the transmission line while the sample is finite in the direction parallel to the transmission line. A rectification algorithm can be used to extract the effective permittivity from the simulated S11 and S21 results [17]. Using the same data as in section A, the effective $\varepsilon$ and $\mu$ of the structure is as shown in Fig. 2. The average $\varepsilon_{\text {eff }}$ from the simulations is 4.4 . This value compares very well with the values from the theoretical equations, and shows that the results from simulations and the inversion process can be used to acquire the effective EM properties of the heterogeneous medium.

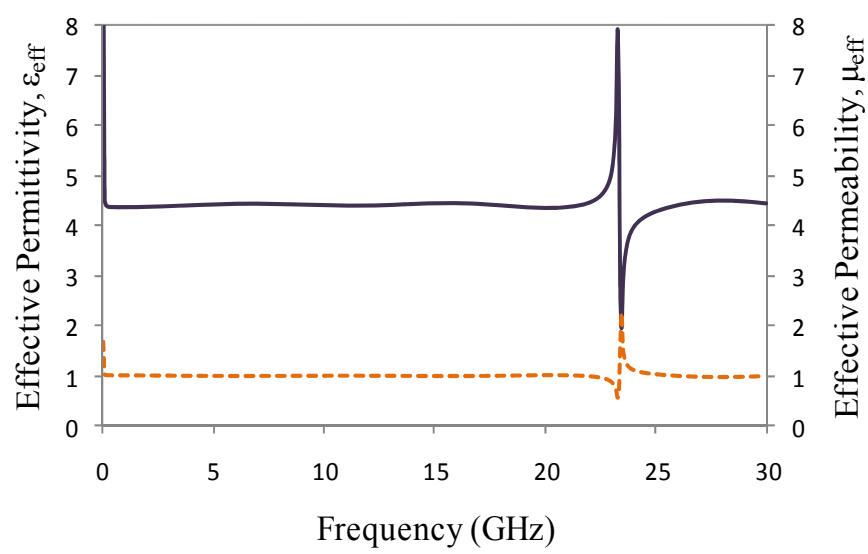

Fig. 2. Variation of effective $\varepsilon(-)$ and $\mu(--)$ of a heterogeneous medium with frequency for $50 \mu \mathrm{m}$ spheres, using simulations via inversion.

The spikes shown in Fig. 2 at $\sim 23 \mathrm{GHz}$ reflect the measurement uncertainty in the extraction of the effective parameters due to the thickness resonance of the structure at that frequency. 


\section{METALLIC INCLUSIONS}

The theoretical and FDTD processes have been extended to the case where the inclusions are metallic. In order to obtain an accurate comparison between theory and simulations, the Drude model was used to obtain the dielectric constant, $\varepsilon_{r}$ of a metal inclusion, in this Copper $(\mathrm{Cu})$, see equation [19].

$$
\varepsilon_{r}=\varepsilon_{d}-\frac{\omega_{p}}{(2 \pi f)^{2}+v_{e}^{2}}\left(1+j \frac{v_{e}}{2 \pi f}\right)
$$

where $\omega_{p}=\frac{N(c h)^{2}}{\varepsilon_{0} m_{e}}$ is the plasma angular frequency, $\varepsilon_{d}$ is the dark dielectric constant, $v_{e}$ is the collision angular frequency, $m_{e}$ is the mass of the electron, $c h$ is the electron charge, $\varepsilon_{0}$ is permittivity of free space, $f$ is frequency. In our calculations, $\varepsilon_{d}$ was chosen arbitrarily as its value is usually too small to have a significant effect on the second term of the equation. For Copper, $m_{e}=1.3 m_{0}, m_{0}$ is free electron mass, $v_{e}=4.23 \times 10^{13} \mathrm{~s}^{-1}$. Using (2) gave $\varepsilon_{r}=(1.27-\mathrm{j} 103.53) \times 10^{6}$, at $10 \mathrm{GHz}$.

These values of permittivity allowed us use the canonical equations in instances where Copper particles were the inclusions. Due to this very high value of $\varepsilon_{2}$ (Copper inclusions), the theoretical maximum by which the effective permittivity of a host medium can be increased by is four times [1]. The effective permittivity of a heterogeneous medium with metallic inclusions is significantly dependent on the volume fraction of the inclusions.

\section{A. Non-Uniform Lattice}

In this section, thin metallic inclusions are placed in a nonuniform lattice. At the end of this section the results are compared to spheres in a cubic lattice with the same equivalent volume fraction.

Investigating non-uniform lattices will increase the range of applicable fabrication techniques. Given that spherical inclusions would be difficult to reproduce in the laboratory for initial measurements, the next stage in our research was to study the effective permittivity of Copper squares etched onto a readily-available dielectric such as GTS. GTS has a relative permittivity of 3 and a standard thickness of $125 \mu \mathrm{m}$. The thickness of the cuboids is $35 \mu \mathrm{m}$. The $\mathrm{Cu}$ 'cuboids' measure $500 \mu \mathrm{m} \times 500 \mu \mathrm{m} \times 35 \mu \mathrm{m}$, spaced $1000 \mu \mathrm{m}$ apart in the $\mathrm{Y}$ and $Z$ axes, and separated by the GTS thickness in the $X$ axis. Thus the periodicity of the structure is $1000 \mu \mathrm{m}$ in the $\mathrm{Y}$ and $\mathrm{Z}$ directions and $195 \mu \mathrm{m}$ in the $\mathrm{X}$ direction. This geometry is based on sizes and materials that could be easily etched.

By stacking a number of layers together as shown in Fig. 3, we can understand how the total structure's effective permittivity changes with the number of layers, and see if it matches the value from the canonical equations when an equivalent volume fraction is used to approximate a periodic structure.

The stacking gives the $\mathrm{Cu}$ a $70 \mu \mathrm{m}$ thickness. Fig. 3 shows a 4-layer structure simulation set-up using the FDTD technique mentioned in Section II. The air gaps between the stacks has an effect on the value of the host permittivity, $\varepsilon_{1}$.

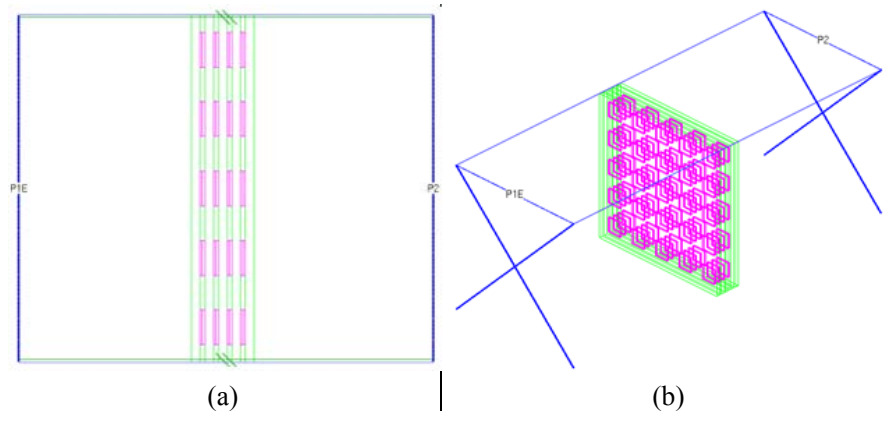

Fig. 3. (a) 2D and (b) 3D representation of a 4-layer stack of heterogeneous medium with $35 \mu \mathrm{m}$ thick Cu etchings on $125 \mu \mathrm{m}$ GTS substrate

As shown in Fig. 4, as the number of layers increase, the effective permittivity of the structure tends towards a certain value. The 12 and 14-layer values are significantly different from the 2-layer value. From previous work [16], we have found that thin samples do not produce the expected results and typically the accuracy of the simulations and the related effective permittivity extraction method increases with thicker samples.

From Fig. 4, as the number of layers increases, the effective permittivity of the dielectric/copper composition converges to an average value of 3.81. By using the volume of the $\mathrm{Cu}$ cuboids to find an equivalent sphere radius, and the $\mathrm{Cu}$ 's volume fraction to obtain approximate cubic lattice spacing, the stacked layer structure can be equated to spheres evenly spaced in a cubic lattice which in turn allows comparison with the theoretical equations. These calculations give an equivalent sphere radius of $161.06 \mu \mathrm{m}$ and cubic spacing of $579.89 \mu \mathrm{m}$. The results from the equations which give an $\varepsilon_{e f f}$ of 3.89. The results from the FDTD simulation of these sphere give an $\varepsilon_{e f f}$ of 3.91 as shown in Fig. 5. These results indicate there is good agreement between the non-uniform stack using the FDTD method and the equivalent structure composed of spheres in a cubic uniform lattice using both the empirical equations and also the FDTD method.

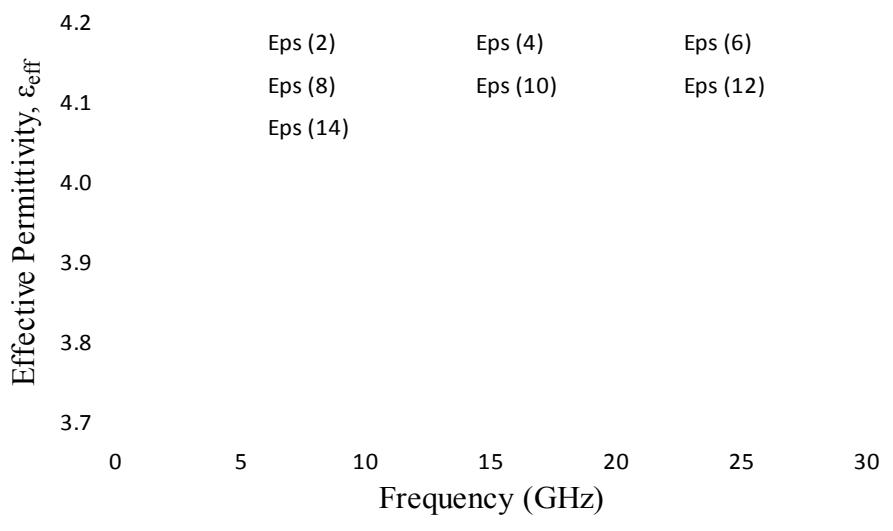


Fig. 4. Variation of $\varepsilon_{e f f}$ with frequency of an n-layer heterogeneous structure having $35 \mu \mathrm{m}$ thick $\mathrm{Cu}$ etchings on a GTS substrate

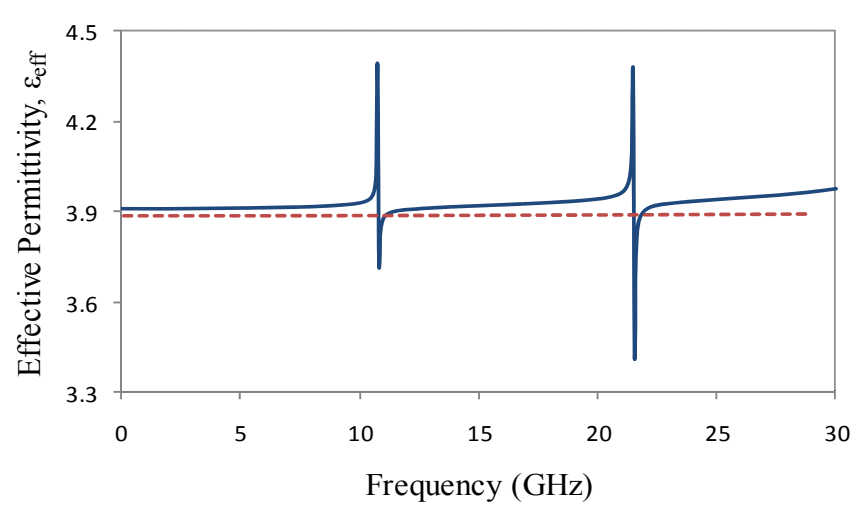

Fig. 5. Variation of $\varepsilon_{e f f}$ with frequency of spheres in a cubic lattice with the equivalent volume ratio to the heterogeneous copper stack structure in Fig. 5 The solid blue line is from the EM simulations while the dotted red line is from the theoretical equations.

\section{CONCLUSIONS}

The canonical equations were found to agree well with EM simulations and the rectification algorithm when spheres were positioned in a uniform lattice. This geometry is reasonably well understood, however there is uncertainty as to the electromagnetic behavior when the inclusions are not spherical and are not evenly spaced. A structure consisting of metallic inclusions in a non-uniform lattice has been examined in this paper. The results showed good agreement with the equivalent volume fraction using spheres in a cubic uniform lattice. The results in this paper show that different inclusion geometries in non-uniform lattices will produce similar results and also that the inclusion permittivity and the volume filling ratio are the dominant parameters to determine the effective permittivity. These results indicate that we can design and fabricate our heterogeneous substrates using different geometries and that we can initially estimate the effective permittivity of a mixture by using the canonical equations. Note, higher effective permittivities can be produced using higher volume fractions of the inclusions. Future work will design geometries that will produce greater contrasts between the effect and the host permittivity and hence will facilitate comparison with measurements. Experimentally, it is possible to fabricate nanomaterials samples where the size of embedded inclusions can be varied from the nm range up to the level of mm, while their spatial arrangement can vary from a high degree of order to randomness.

\section{REFERENCES}

[1] L. Lewin, "The electrical constants of a material loaded with spherical particles", IEE-Part III: Radio Comm. Eng., vol. 94, issue 27, pp. 65-68, Jan. 1947.

[2] L. Rayleigh, "On the influence of obstacles arranged in rectangular order on the properties of a medium", Phil. Mag., series 5, vol. 34, pp. 481-502, 1892
[3] J. Brown, "Artificial dielectrics having refractive index less than unity", Proc. IEE-Part IV: Inst. Mono,, vol. 100, issue 5, pp. 51-62, Sept. 1953

[4] W. T. Doyle, "The Clausius-Mossotti problem for cubic array of spheres", J. App. Phys., vol. 49, issue 2, pp. 795-797, Feb. 1978.

[5] X. Cai, R. Zhu, and G. Hu, "Experimental study for metamaterials based on dielectric resonators and wire frame", Metamaterials, vol. 2, issue 4, pp. 220-226, Sept. 2008.

[6] W. T. Doyle, "Optical properties of a suspension of metal spheres", Phy. Rev. B, vol. 39, issue 14, pp. 9852-9858, May 1989.

[7] R. E. Collin, Field Theory of Guided Waves, IEEE Press: NY, 1991, ch. 12.

[8] A. Sihvola, Electromagnetic Mixing Formulas and Applications, IEE Elect. Waves Series 47, 1999, ch. 3 .

[9] I. A. Kolmakov, L. Jylha, S. A. Tretyakov, and S. Maslovki "Lattice of dielectric particles with double negative response", $28^{\text {th }}$ Gen. Ass. Int. Union Radio Sci. (URSI), paper BCD.5 (0109).pdf, 2005

[10] C. L. Holloway, E. F. Kuester, J. B-Jarvis, and P. Kabos, "A double negative (DNG) composite medium composed of magnetodielectric spherical particles embedded in a matrix", IEEE Trans. Antennas Propagat., vol. 51, no. 10, pp. 2596-2603, Oct. 2003.

[11] W. G. Whittow, and J. C. Vardaxoglou, "Modifying conventional microwave antenna designs using fine scale structures and nanomaterials", LAPC 2009, pp. 749-752, Dec. 2009.

[12] F. Juillerat, H. H. Solak, P. Bowen, and H. Hofmann, "Fabrication of large-area ordered arrays of nanoparticles on patterned substrates", Inst. Of Phys. Pub., Nanotech., vol. 16, no. 8, pp.1311-1316, June 2005.

[13] C.-C. Hsu, and Y.-C. Lee, "Fabrication of flexible nano-wired polarizer by contact-transferred and mask embedded lithography and polyurethane acrylate method", Proc. $5^{\text {th }}$ IEEE Int. Conf. NEMS, pp. 893-897, Sept. 2010.

[14] Y. H. Seo, D.-S. Choi, and K.-H. Whang, "A new fabrication method of nano-fluidic filters using self-assembly of nano-spheres and surface tension", $19^{\text {th }}$ Proc. IEEE Int. Conf. MEMS, pp.306-309, May 2006.

[15] C. C. Njoku, W. G. Whittow, and J. C. Vardaxoglou, "Comparative study of nanomaterials' effective properties using canonical equations", LAPC 2010, pp. 413-416, Nov. 2010.

[16] N. G. Alexopoulos, C. A. Kyriazidou, and H. F. Contopanagos, "Effective parameters for metamorphic materials and metamaterials through a resonant inverse scattering approach", IEEE Trans. Microw. Theory Tech., vol. 55, no. 2, pp. 254-267, Feb. 2007.

[17] C. C. Njoku, W. G. Whittow, and J. C. Vardaxoglou, "Study on the variation in dielectric properties of heterogeneous substrates composed of nanomaterials", EuCAP 2011, pp. 488-492, May 2011.

[18] M. A. Ordal, et al., "Optical properties of the metals Al, Co, Cu, Au, Fe, $\mathrm{Pb}, \mathrm{Ni}, \mathrm{Pd}, \mathrm{Pt}, \mathrm{Ag}, \mathrm{Ti}$, and $\mathrm{W}$ in the infrared and far infrared", Applied Optics, vol. 22, no. 7, April 1983.

[19] D. S. Lockyer, J. C. Vardaxoglou, and M. J. Kearney, "FSS array generation by optical means",

[20] I. Awai, Y. Maegawa, and T. Ishizaki, "Measurement of effective material constants of artificial dielectrics made of spherical metal particles", APMC 2009, pp. 1655-1658, Jan. 2010.

[21] K. L. Kelly, E. Coronado, L. L. Zhao, and G. C. Schatz, "The optical properties of metal nanoparticles: the influence of size, shape, and dielectric environment", J. Phys. Chem. B, vol. 107, pp. 668-677, 2003.

[22] O. Rybin, "Enhancement of dielectric constant in metal-dielectric metamaterials", Afr. Phys. Rev. vol. 3, pp. 49 -55, 2009.

[23] J. A. Stratton, Electromagnetic Theory, New York: McGraw-Hill Book Inc., 1941, ch. 9.

[24] K. K. Karkkaninen, A. H. Sihvola, and K. I. Nikoskinen, "Effective permittivity of mixtures: Numerical validation by the FDTD method", IEEE Trans. Geos. Remote Sensing, vol. 38, no. 3, pp. 1303-1308, May 2000.

[25] W. B. Weir, "Automatic measurement of complex dielectric constant and permeability at microwave frequencies", Proc. IEEE, vol. 62, no. 1, pp. 33-36, Jan. 1974

[26] D. R. Smith, D. C. Vier, Th. Koschny, and C. M. Soukolis, "Electromagnetic parameter retrieval from inhomogeneous metamaterials", Phys. Rev. E71, 036617, 2005. 